This item was submitted to Loughborough's Research Repository by the author.

Items in Figshare are protected by copyright, with all rights reserved, unless otherwise indicated.

\title{
Compressed sensing current mapping methods for PV characterisation
}

PLEASE CITE THE PUBLISHED VERSION

https://doi.org/10.1109/PVSC.2016.7749827

PUBLISHER

(c) IEEE

VERSION

AM (Accepted Manuscript)

LICENCE

CC BY-NC-ND 4.0

REPOSITORY RECORD

Koutsourakis, George, Matt Cashmore, Martin Bliss, Simon R.G. Hall, Thomas R. Betts, and Ralph Gottschalg. 2019. "Compressed Sensing Current Mapping Methods for PV Characterisation". figshare.

https://hdl.handle.net/2134/22058. 


\title{
Compressed Sensing Current Mapping Methods for PV Characterisation
}

\author{
George Koutsourakis ${ }^{1}$, Matt Cashmore ${ }^{2}$, Martin Bliss ${ }^{1}$, Simon R. G. Hall ${ }^{2}$, Thomas R. Betts ${ }^{1}$, and Ralph \\ Gottschalg ${ }^{1}$. \\ ${ }^{1}$ Centre for Renewable Energy Systems Technology (CREST), Loughborough University, Loughborough, \\ Leicestershire, LE11 3TU, United Kingdom \\ ${ }^{2}$ National Physical Laboratory (NPL), Hampton Road, Teddington, Middlesex, TW11 0LW, United \\ Kingdom
}

\begin{abstract}
The Compressed Sensing (CS) sampling theory has been combined with the Light Beam Induced Current (LBIC) method, to produce an alternative current mapping technique for photovoltaic (PV) devices. Compressive sampling of photocurrent is experimentally implemented using a Digital Micro-mirror Device (DMD). The main advantage of this new method for current mapping is that measurement time can be significantly reduced compared to conventional LBIC measurement systems. This is achieved mainly by acquiring fewer measurements than a raster scan would need and by utilizing the fast response of the micro-mirror array. Two different experimental layouts are considered in this work. The first is a small area optical set-up based on a single wavelength laser source. The second layout utilizes a commercial Digital Light Processing (DLP) projector through which compressive sampling is applied. Experimental results with both experimental schemes demonstrate that current maps can be produced with less than $50 \%$ of the measurements a standard LBIC system would need. The ability to acquire current maps of individual cells in encapsulated modules is also highlighted. The advantages and drawbacks of the method are presented and its potential to significantly reduce measurement time of current mapping of PV cells and modules is indicated.
\end{abstract}

Index Terms - compressed sensing, PV cell testing, current mapping, PV characterisation

\section{INTRODUCTION}

Spatial characterization of photovoltaic (PV) devices is essential and leads to an improvement of PV product quality through detection and prevention of production induced defects. There are several established spatially resolved PV characterisation methods that can provide a number of performance parameters of PV devices. These techniques include electroluminescence [1], photoluminescence [2], lockin thermography [3] and light beam induced current (LBIC) measurements [4].

Photocurrent mapping provides insight of local optical and electrical parameters of PV devices, as well as a better understanding of their material and degradation properties. Quantitative current mapping is usually achieved by Laser/Light Beam Induced Current (LBIC) measurements. The standard methodology for an LBIC system consists of a light beam that scans the PV sample while the induced current is measured at every point to acquire the final current map. The most significant drawback of such techniques is that measurements are very time consuming. Measurements often last several hours in order to produce a high resolution current map. Additionally, experimental layouts can be rather complicated and cumbersome as they may require lock-in amplifiers and bias lighting.

In this work new experimental schemes for current mapping of PV devices are explored in order to significantly reduce measurement time. The applied method is based on the compressed sensing (CS) sampling theory [5][6]. According to the CS theory, one can reconstruct a signal from highly incomplete or inaccurate information. Compressed sensing uses a similar type of mathematics as JPEG compression does for storing images. In JPEG image compression, most of the information of an image is discarded at the compression stage. Only $\mathrm{K}$ elements are kept, while the image can be accurately restored using these $\mathrm{K}$ elements. Similarly, the aim of CS imaging is to directly acquire the $\mathrm{K}$ coefficients necessary for an almost exact reconstruction of a signal or an image. This is achieved by only acquiring $\mathrm{M}<<\mathrm{N}$ linear measurements for capturing an $\mathrm{N}$ pixel image, where $\mathrm{K}<<\mathrm{M}$.

A compressed representation of a sparse signal $\mathbf{x}$ can be acquired using $\mathrm{M}<\mathrm{N}$ linear measurements between $\mathbf{x}$ and a collection of test functions $\Phi=\left\{\varphi_{\mathrm{m}}\right\}_{\mathrm{m}=1}^{\mathrm{M}}$. This means that a convex optimization problem is created by measuring $\mathbf{y}[\mathrm{m}]=\left\langle\mathbf{x}, \varphi_{\mathrm{m}}\right\rangle$. Minimization of the $\ell 1$ norm is used to find the solution: $\hat{\mathbf{x}}=\operatorname{argmin}\|\mathbf{x}\|_{1}$ subject to $\Phi \mathbf{x}=\mathbf{y}$. For the realization of CS current mapping measurements, a series of random patterns is projected on the sample under test. The current response is measured for each pattern. These patterns correspond to the test functions required for compressive sampling. The final current map is acquired via a reconstruction algorithm. A Digital Micromirror Device (DMD) is used [7] for the generation of the patterns. The DMD array has a very short response time, which is less than $20 \mu \mathrm{s}$. Combined with the reduced number of measurements needed to acquire the complete current map, measurement time can be reduced by at least an order of magnitude. Moreover, it has been demonstrated already that point by point current mapping utilizing a DMD chip [11] or a DLP projector [12] is feasible. 
Two different experimental CS current mapping schemes are considered in this work. A CS current mapping optical prototype has been built at NPL based on a DMD chip. This initial setup can perform small area scans and initial measurements have proved the feasibility of the method [8][9]. Current maps have been produced with just $30 \%$ of the measurements a standard LBIC system would need. As a scaleup of the small area system, a larger setup based on a digital light processing (DLP) projector has been built at CREST. The aim is to explore the possibility of utilizing projection technology for applying CS current mapping measurements in larger PV devices. The major advantage of using projection technology is that individual cells in encapsulated modules can be assessed. The cell under test can be current limited by adjusting the necessary CS patterns, while the rest of the cells are light biased [10].

\section{EXPERIMENTAL SYSTEMS}

\section{A. Prototype small area experimental setup}

A prototype has been built in NPL for the realization of small area CS current mapping of PV devices. Two different light sources have been used, a $40 \mathrm{~mW} 658 \mathrm{~nm}$ laser and a $100 \mathrm{~mW}$ $785 \mathrm{~nm}$ laser. After exiting a single mode fiber, the beam is expanded and collimated, overfilling the DMD chip. The DMD generates the series of patterns to be projected on the sample under measurement. The DMD array has a $1024 \times 768$ resolution, with each micromirror having a side length of approximately $14 \mu \mathrm{m}$. Thus, the DMD area is approximately $1.4 \mathrm{~cm}$ by $1 \mathrm{~cm}$. A $768 \times 768$ pixel area of the DMD is utilized resulting in a maximum measurement area of approximately $1 \mathrm{~cm}$ by $1 \mathrm{~cm}$. The projection is currently not magnified. A spatial filter is used for a cleaner projected image. A schematic diagram of the setup is presented in Fig. 1.

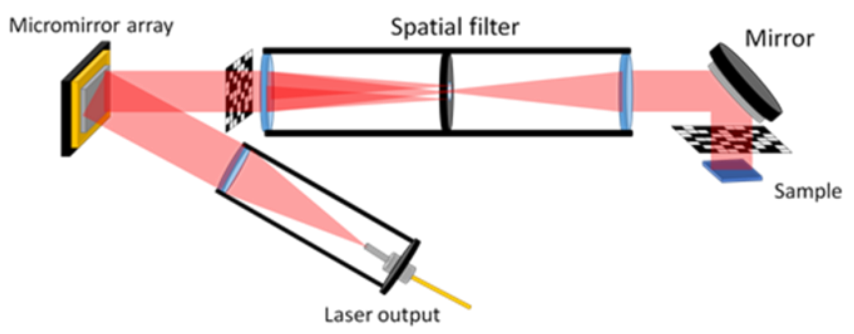

Fig. 1. Schematic diagram of the small area CS current mapping prototype built at NPL.

The discrete cosine transform is applied as a basis to provide the sparse representation of the signal. Random binary matrices are used for sampling in this system, to produce the sensing matrix [13]. Reconstruction of the final current map is realised in MatLab, using the $\ell 1$ MAGIC package [14]. A significant advantage of using the DMD is that its switching response time is shorter than $20 \mu \mathrm{s}$, which results in increased measurement speed. Ultimately, measurement speed is limited by the sampling rate and the number of samples taken for each pattern. In this series of measurements the speed was approximately 10 samples per second, but there is potential for faster sampling with software and hardware optimisation. Since the patterns illuminate around half of the cell each time, the high signal to noise ratio diminishes the need for a lock-in amplifier. 100 by 100 pixel images with $50 \%$ of measurements acquired (5000 patterns) are generated in approximately 8 minutes.

\section{B. DLP projector based system}

For realizing CS current mapping of larger area PV devices and especially PV modules, another scheme is explored utilizing a DLP projector. An ACER 7605 DLP projector is used in this work. The projector has a $370 \mathrm{~W}$ Osram UHP lamp, which provides a light output of 5000 lumens. The internal 1920x1200 pixel DMD chip creates the projection's patterns. The key advantage of DLP technology is the high contrast ratio. It allows the system to generate a black pixel equivalent to a masked shaded spot on the PV module. A drawing of the experimental setup is presented in Fig. 2. All measurements were made in a dark room, to minimize background light influence.

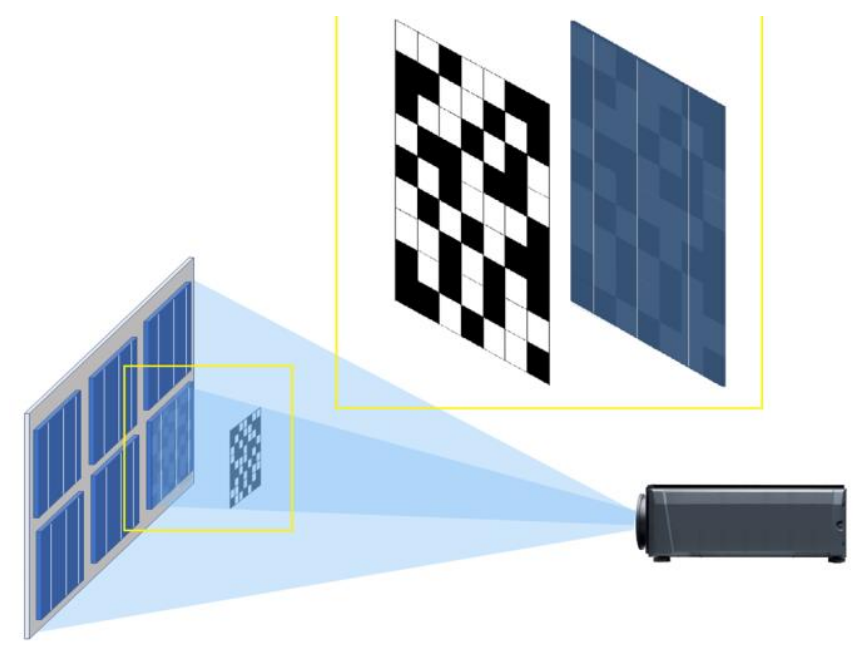

Fig. 2. Drawing of the DLP projector based experimental layout for CS current mapping.

Prior to measurements, the projector's properties were investigated, as the light intensity is expected to vary temporally and spatially. Due to the internal colour wheel synchronized with the DMD chip of the projector, the colours are displayed sequentially at a high rate such that only the final combination of colours can be observed by the human eye. This operation may be well suited for usual projection requirements, but creates significant spectral and temporal variations of irradiance for PV characterisation. In order to overcome this limitation, the colour wheel of the projector was removed from the light path. As a result the projector only projects in black 
and white. The spectrum is stable during measurements. This approach would provide a very simple and cost-effective spatial characterisation tool and make the first step towards a dedicated projector system for PV characterisation.

For the case of series interconnected PV modules, the patterns are projected on the cell under measurement while the remaining cells are light biased. This is necessary to ensure the cell under measurement is the limiting cell of the module. As there are no bypass diodes, the current measured at the contacts of the module is the current of the cell under test [10]. Current maps of fully encapsulated modules can be acquired with this simple setup.

Although the projector is controlled through a LabView environment, there is not direct control of the internal DMD chip of the commercial projector. The micromirrors that create the projections still turn on and off independently at a rate faster than $20 \mu \mathrm{s}$, inserting temporal variations of irradiance on the sample. Fortunately these variation are periodic, with a period of approximately $4 \mathrm{~ms}$. Thus, current readings were acquired with an integration time of multiples of this period, to minimize measurement noise. Another issue is variation of the lamp intensity at random time intervals. Constant monitoring of the light intensity and calibration of each measurement is necessary to minimize the influence of this issue.

For this series of measurements with the projector system, the standard laminate size modules used for ageing experiments at CREST are used. These are 6-cell crystalline Silicon (c-Si) mini modules which are produced in-house. Each cell has a size of $15.7 \mathrm{~cm}$ by $15.7 \mathrm{~cm}$. The terminals of each individual cell were extended to the outside of the encapsulation, allowing direct contact of each individual cell for validation purposes. This alternative operation allows a robust validation of measurements results, but also the possibility of CS current mapping of individual cells. No by-pass diodes are integrated within the mini-module.

\section{MEASUREMENT RESULTS}

In Fig. 3, CS current mapping results of a $1 \mathrm{~cm}$ by $1 \mathrm{~cm}$ area of a c-Si cell are presented. An EL image of the c-Si sample used is also included for comparison. The current map size is 96x96 pixels, as a result the optical resolution is approximately $100 \mu \mathrm{m}$. The current maps were reconstructed acquiring different numbers of measurements and are presented as a percentage of the total number of pixels of the current map (i.e. $100 \%$ being the number of measurements a raster scan would need). In the case presented here, the total number of pixels is 9216. As an example, the $30 \%$ current map was produced with 2765 measurements.

It is evident from the results of Fig. 3 that significantly fewer measurements are needed for acquiring a current map than when using a standard LBIC point by point scan. A CS current mapping system requires less than half of the time a standard LBIC system needs to scan the same area with the same resolution. Moreover, as half of the area of the sample is illuminated rather than only one point, the signal to noise ratio is greatly enhanced, eliminating the need for lock-in techniques. This simplifies and accelerates the measurement. Results show that from over $40 \%$ (3686) measurements, there is no significant further improvement of the current map.
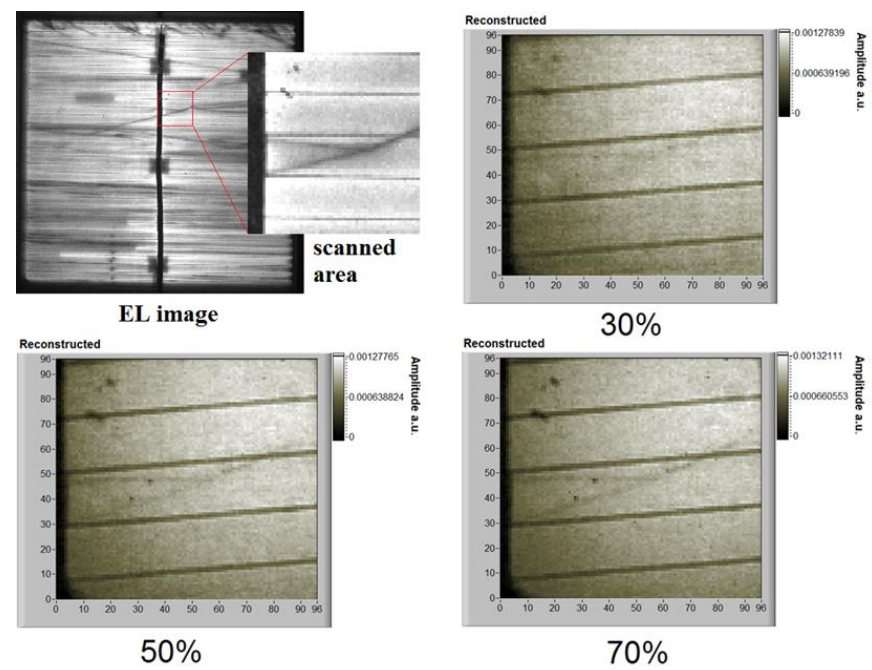

Fig. 3. CS current mapping measurement results of a $1 \mathrm{~cm} \times 1 \mathrm{~cm}$ area of the mc-Si sample, with different number of acquired measurements. The EL image is also presented (top left) for comparison.

The Gaussian top-hat distribution of the irradiance of the beam is obvious in the current maps generated by the small area setup. In addition, the projection plane does not exactly coincide with the plane of the sample, thus the image appears as if it is observed at an angle. In future implementations a diffuser with additional optics can be used before beam collimation for a more uniform intensity of the projection.

Using the DLP projector for CS current mapping is not as straightforward as the small area layout. Temporal variations of light intensity introduce considerable measurement noise that affects compressive sampling accuracy. Additionally, the broad spectrum of the incident light is not optimal for current mapping of crystalline silicon devices. The same mathematical procedure as in the small area setup case is used for applying compressive sampling. Current maps of a single c-Si cell were acquired to test the functionality of the system (Fig. 4). The percentage is the ratio of measurements acquired, to the total number of pixels of the current map. Random patterns are used for compressive sampling, while the $\ell 1$ magic solver is used for reconstruction.

Although the resolution is not high enough to distinguish the fingers of the cell, the busbars are clearly visible, which shows that the method is feasible. In addition, as the pattern again illuminates half of the cell, high signal to noise ratio is achieved. Above $80 \%$ of measurements, the increased noise of 
the system starts having an effect on reconstruction results, corrupting the current map. However, the purpose of the method is to acquire a smaller number of measurements, so the reconstructed current maps in the range of $40 \%$ to $60 \%$ of measurements provide the best results.
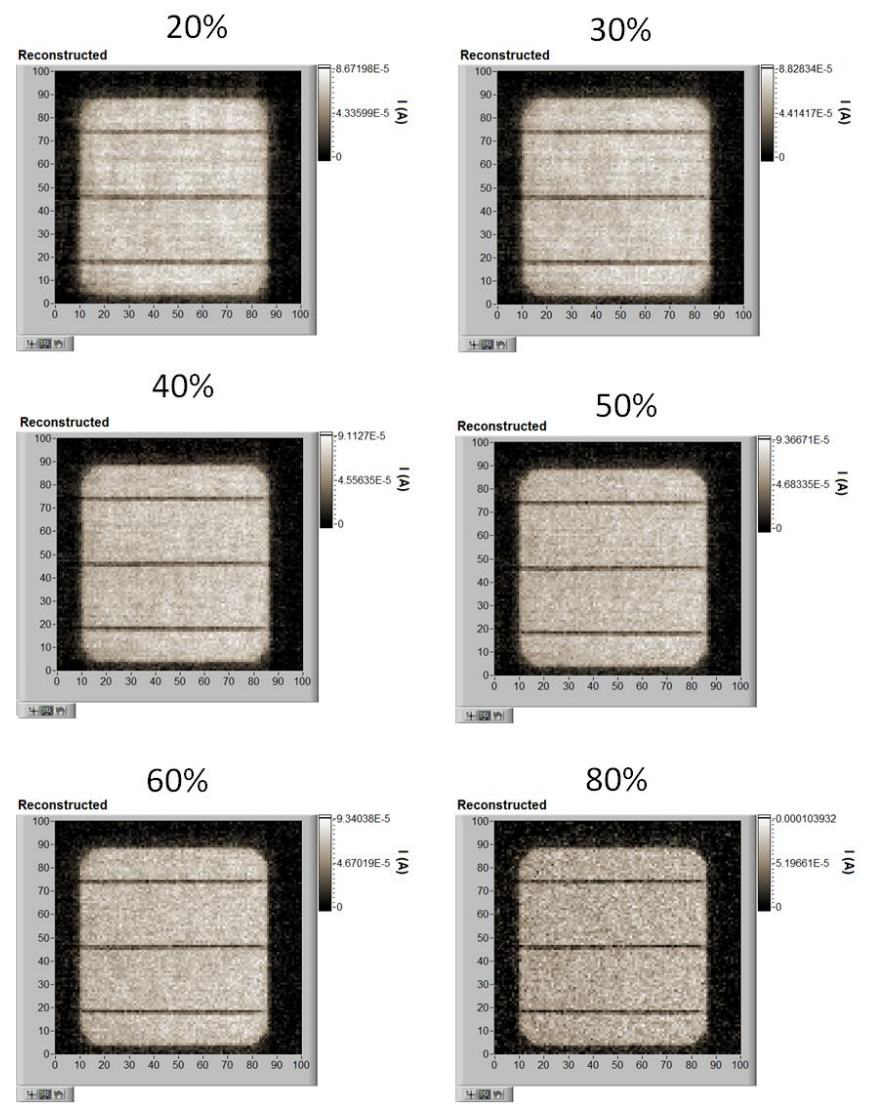

Fig. 4. CS current maps of a single c-Si cell. Number of measurements is expressed as a ratio of number of measurements acquired by the number of pixels of the images.

The next step is to test if CS current mapping of PV modules is possible with such a simple system based on a DLP projector. The module used consisted of c-Si cells of the same type, as the one presented above. Each cell was sampled with the necessary patterns consecutively, while the rest were fully illuminated, as illustrated in Fig. 2. This is extremely easy to achieve using a DLP projector. Current mapping of PV modules requires shading the cell under measurement for limiting and light biasing the rest. A DLP projection system provides the means to accomplish this, without the need of any mechanical shading mechanisms. The resolution achieved in these results is approximately $1.5 \mathrm{~mm}$.

A reconstructed CS current map of the 6-cell PV module is presented in Fig.5. 50\% of measurements were acquired, compared to the pixels of the current map. Results still contain a significant amount of noise, due to the temporal variations of light intensity. Due to the large integration time for every measurement, total sampling time was 6 hours (1 hour for each cell). Reconstruction time is approximately one minute, which is a negligible amount compared to actual measurement time

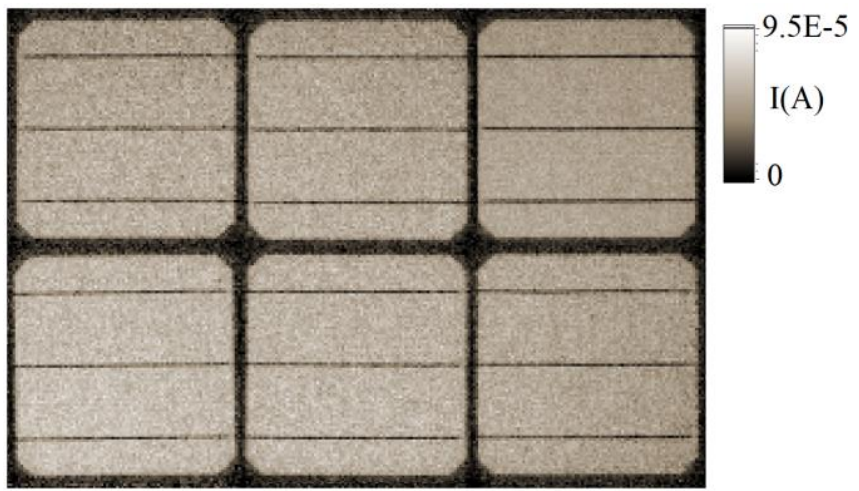

Fig. 5. CS current map of the 6 cell PV module used in these experiments. The CS current map is produced having acquired 30000 $(50 \%)$ of measurements.

CS current mapping with the DLP projector system was also realised for a thin film PV device. An a-Si thin film mini module with a size of $5 \mathrm{~cm}$ by $5 \mathrm{~cm}$, consisting of 3 series connected cells was used for measurements. The same principles as in the case of the c-Si modules were followed, but this time the patterns were not square but rectangular, in order to cover individual cells of the mini module, as shown in the photo on the left side of Fig. 6. A resolution of 10000 pixels was used for each cell. The results (right side of Fig. 6) indicate that CS current mapping with a DLP projection technology can also work for thin film modules, using the same methodology of light biasing for the cells not under measurement.

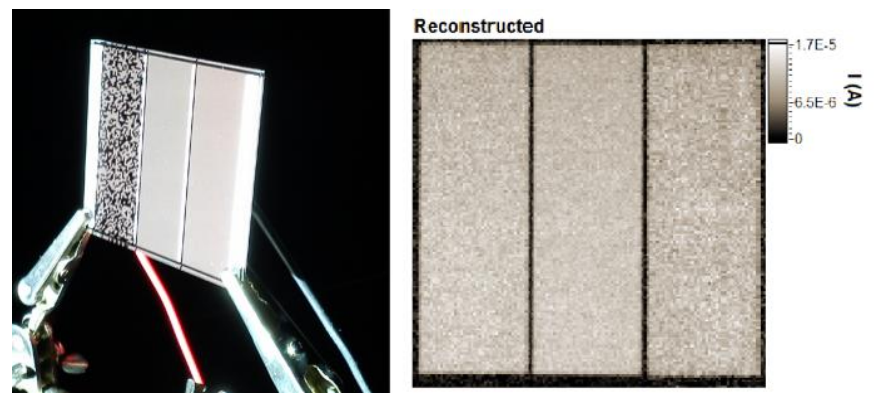

Fig. 6. CS current map of the 6 cell PV module used in these experiments. The CS current map is produced acquiring $50 \%$ of the measurements over a total count of 30000 pixels.

In the current system there are high noise levels evident in the current maps. This is due to the commercial projector the system is based on. However, the results indicate that low resolution measurements are possible even with such a system. A custom made DLP projection based experimental layout for CS current mapping is necessary for optimized measurements. 
This must include a single wavelength temporally stable light source, direct control of the DMD sub-system and the equivalent projection optics of a projector. Measurement speed will be significantly increased with such a dedicated system, reaching and likely exceeding the speed of the small area setup, due to the high signal to noise ratio that can be achieved. The same optical principles used in DLP projectors will be applied, but a single wavelength high power light source should be of more interest, for more accurate determination of penetration depth into the PV material.

\section{CONCLUSIONS}

An alternative approach for current mapping of PV devices is presented in this work. The technique is based on the compressed sensing sampling theory and can significantly reduce the measurement time of current mapping for PV device characterization. Instead of a point by point scan, a series of random patterns is projected on the sample under test, measuring the current response for each pattern and building the final current map using an optimization algorithm. The measurements required are much fewer than when applying a raster scan, which results in a reduced measurement time for current mapping. Further advantages are high signal to noise ratio for current readings and much simpler experimental layouts compared to standard LBIC systems.

The method is experimentally demonstrated through two different schemes. An experimental setup for small area measurements based on a DMD chip has demonstrated that the technique is feasible and reliable current maps can be acquired with much fewer measurements than what a standard LBIC system would need. A second approach is presented, utilizing a DLP projector for creating the necessary patterns for CS current mapping. Experimental results demonstrate that a CS current mapping system based on DLP projection technology could provide a realistic solution for current mapping of PV modules. However, the prototype system of this work cannot achieve very high accuracy and resolution by using a standard commercial DLP projector. A custom system would provide more control and be able to reduce temporal variations of light intensity and consequently provide higher accuracy.

\section{ACKNOWLEDGMENTS}

This work was funded through the European Metrology Research Programme (EMRP) Project ENG55 PhotoClass. The EMRP is jointly funded by the EMRP participating countries within EURAMET and the European Union. This work is cofunded by the UK National Measurement System.

\section{REFERENCES}

[1] T. Fuyuki, H. Kondo, T. Yamazaki, Y. Takahashi, and Y. Uraoka, "Photographic surveying of minority carrier diffusion length in polycrystalline silicon solar cells by electroluminescence," Appl. Phys. Lett., vol. 86, no. 26, p. 262108, 2005.

[2] T. Trupke, B. Mitchell, J. W. Weber, W. McMillan, R. a. Bardos, and R. Kroeze, "Photoluminescence Imaging for Photovoltaic Applications," Energy Procedia, vol. 15, no. 2011, pp. 135-146, Jan. 2012.

[3] O. Breitenstein, J. P. Rakotoniaina, and M. H. Al Rifai, "Quantitative evaluation of shunts in solar cells by lock-in thermography," Prog. Photovoltaics Res. Appl., vol. 11, no. 8, pp. 515-526, Dec. 2003.

[4] M. Padilla, B. Michl, B. Thaidigsmann, W. Warta, and M. C. Schubert, "Short-circuit current density mapping for solar cells," Sol. Energy Mater. Sol. Cells, vol. 120, pp. 282-288, Jan. 2014.

[5] D. Donoho, "Compressed sensing," Inf. Theory, IEEE Trans., vol. 52, no. 4, pp. 1289-1306, 2006.

[6] E. Candes, J. Romberg, and T. Tao, "Stable signal recovery from incomplete and inaccurate measurements," Comm. Pure Appl. Math., vol. 59, pp. 1207-1223, 2006.

[7] L. Hornbeck, "The DMDTM projection display chip: a MEMSbased technology," Mrs Bull., vol. 26, no. 4, pp. 325-327, 2001.

[8] S. R. G. Hall, M. Cashmore, J. Blackburn, G. Koutsourakis, and R. Gottschalg, "Compressive Current Response Mapping of Photovoltaic Devices using MEMS mirror arrays," IEEE Trans. Instrum. Meas., 2016.

[9] G. Koutsourakis, X. Wu, M. Cashmore, S. R. G. Hall, M. Bliss, T. R. Betts, and Gottschalg, "Fast Current Mapping of Photovoltaic Devices Using Compressive Sampling," 31st EUPVSEC Proc., pp. 29 - 34, 2015.

[10] P. Vorasayan, T. R. Betts, and R. Gottschalg, "Limited laser beam induced current measurements: a tool for analysing integrated photovoltaic modules," Meas. Sci. Technol., vol. 22, no. 8, p. 085702, Aug. 2011.

[11] R. Gupta and O. Breitenstein, "Digital micromirror device application for inline characterization of solar cells by tomographic light beam-induced current imaging," Proc. SPIE, vol. 6616, pp. 66160-1-66160O-9, 2007.

[12] J. Yoo, S. Kim, D. Lee, and S. Park, "Spatial uniformity inspection apparatus for solar cells using a projection display," Appl. Opt., vol. 51, no. 20, pp. 4563-4568, 2012.

[13] R. Baraniuk, M. Davenport, R. DeVore, and M. Wakin, “A Simple Proof of the Restricted Isometry Property for Random Matrices," Constr. Approx., vol. 28, no. 3, pp. 253-263, Jan. 2008.

[14] E. Candès, J. Romberg, and T. Tao, "Robust uncertainty principles: Exact signal reconstruction from highly incomplete frequency information," IEEE Trans. Inf. Therory, vol. 52, no. 2, pp. 489-509, 2006. 